\section{DEVELOPMENT AND VALIDATION OF A FINITE ELEMENT MODEL OF THE PELVIS}

\section{Andrew E. Anderson (1) Christopher L. Peters (2) Benjamin D. Tuttle (1) Jeffrey A. Weiss $(1,2)$}

(1) Department of Bioengineering, University of Utah

(2) Department of Orthopaedics, University of Utah Salt Lake City, Utah

\section{INTRODUCTION}

An improved understanding of the stress distribution in and around the hip joint may provide important information regarding the relationship between altered pelvic and acetabular geometry and development of hip osteoarthritis, as well as point to improved diagnostic methods and analysis of surgical treatment. It is very difficult to accurately assess how changes in pelvic geometry affect the stress and strain distribution of the joint in an experimental setting. The finite element (FE) method provides an alternative approach for study of hip joint mechanics. Although FE models of the pelvis have been developed, validation by direct comparison with subject-specific experimental measurements has not been performed. In addition, previous models have utilized over-simplified bone geometry and homogeneous material properties. The objectives of this study were to 1) develop and validate a FE model of the pelvis using subject-specific measurements of bone geometry as well as location-dependent cortical thickness and trabecular bone elastic modulus, and 2) assess the sensitivity of the subject-specific FE model to changes in material properties and cortical thickness.

\section{METHODS}

A combined experimental and computational protocol was used to develop and validate a subject-specific three-dimensional model of a $68 \mathrm{y} / \mathrm{o}$ female pelvis.

\section{Experimental Study:}

The sacroiliac joint and all soft tissue, with the exception of articular cartilage, were removed. A registration block and wires were attached to the iliac crest. The block allowed for spatial

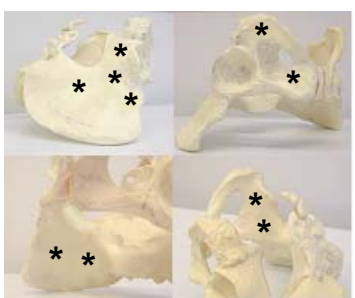

Fig. 1: Model indicating locations of rosette strain gauges used on the cadaveric pelvis. registration of experimental and FE coordinate systems, while the wires served as a guide to reproduce the boundary conditions used in the experimental model. A volumetric CT scan $(512 \times 512$ acq. matrix, $\mathrm{FOV}=225 \mathrm{~mm}, 354$ slices, slice thickness $=0.6 \mathrm{~mm}$ ) was obtained in a superior to inferior fashion.

The iliac crests were submerged in a mounting pan of quicksetting cement to the depth defined by the iliac guide wires. Ten rosette strain gauges (Vishay Measurements Group, Raleigh, NC), representing 30 channels, were attached to the hemi-pelvis at locations around the acetabulum, pubis, ischium, and ilium (Fig. 1). Vertically orientated loads $(0.25,0.50,0.75$, and $1.0 \mathrm{BW})$ were applied to the acetabulum via a femoral prosthesis, attached to a linear actuator, while strains were recorded continuously. The strain gauge data were converted to minimum and maximum in-plane principal strains. 3D coordinates of the strain gauges and registraton block were determined using an electromagnetic digitizer (Immersion Corp., San Jose, CA).

\section{Computational Analysis and Validation:}

Separate surfaces for the outer cortex and the boundary of the cortical and trabecular bone of the pelvis were extracted from the CT data via manual segmentation (Fig. 2, top). A FE model was created from the surfaces, consisting of 30,000 triangular shell elements for cortical bone and 210,000 tetrahedral solid elements for trabecular bone (Fig. 2, bottom). Acetabular cartilage was represented with 500 shell elements at a constant thickness of $2 \mathrm{~mm}$, determined by averaging the distance between the implant and acetabulum in the neutral kinematic position. A novel algorithm was developed to assign a spatially varying cortical shell thickness to the cortical shell elements based on the distances between the two polygonal surfaces.

The femoral implant was modeled as rigid while cortical and trabecular bone were represented as isotropic hypoelastic. Initial material properties for cortical bone were $E=17$ GPa and $v=0.29$ (Dalstra et al., 1995).

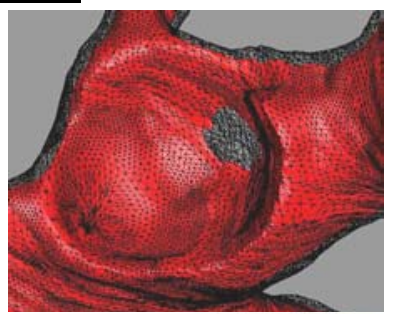
Relationships between CT Hounsfield unit, apparent density and elastic modulus were used to assign a density-dependent modulus for each tetrahedral element (Ploeg et al., 2001; Dalstra et al., 1995). Acetabular articular cartilage was represented as a hyperelastic Mooney-Rivlin material. Coefficients $C_{1}$ and $C_{2}$ for articular cartilage were assumed to be $4.1 \mathrm{MPa}$ and 0.41 $\mathrm{MPa}$ respectively with $\mathrm{v}=0.4$ (Little et al., 1986). Nodes superior to the iliac wires and nodes along the pubis synthesis joint were constrained. Contact was enforced between the femoral implant and cartilage while load was applied to the implant. All analyses were performed with the implicit capabilities of LS-DYNA (Livermore Software Technology Corporation, Livermore, CA). FE predictions of cortical principal strains were averaged over elements that were located beneath each strain gauge.

Sensitivity studies were performed to assess the effects of variations in assumed and measured material properties and cortical thickness on cortical surface strains. The models included (Table 1): constant cortical shell thickness (CST); constant trabecular elastic modulus (CTEM); constant shell thickness and elastic modulus (CST/CTEM); subject-specific with alterations in cortical $v$ (SSCV), trabecular bone $v$ (SSTV), and cortical elastic modulus (SSCM).

Table 1: Models studied for FE sensitivity analysis. Standard deviations (SD) in material properties and cortical thickness were taken from experimentally measured values (EXP) as well as deviations and ranges of values reported in the literature.

\begin{tabular}{|c|c|c|}
\hline Type & Models Analyzed & Reference \\
\hline CST & Thickness $=+/-0,1,2 \mathrm{SD}(0.4 \mathrm{~mm})$ & EXP \\
\hline CTEM & $E=+/-0,0.5,1 \mathrm{SD}(80 \mathrm{MPa})$ & EXP \\
\hline CST/CTEM & Thickness $=1.0 \mathrm{~mm}, \mathrm{E}=160 \mathrm{MPa}$ & EXP \\
\hline SSCV & $\mathrm{v}=0.2, \mathrm{v}=0.39$ & (Lappi et al., 1979) \\
\hline SSTV & $\mathrm{v}=0.29$ & (Oonishi et al., 1983) \\
\hline SSCM & $E=+/-1 \mathrm{SD}(1.62 \mathrm{GPa})$ & (Snyder, Schneider, 1991) \\
\hline
\end{tabular}




\section{RESULTS}

Cortical bone thickness ranged from 0.4-3.0 mm (mean 1.0 \pm 0.4 $\mathrm{mm}$ ). Cancellous bone elastic moduli ranged from 20-400 MPa (mean $160 \pm 80 \mathrm{MPa}$ ). The subject-specific FE model predictions of principal strains (Fig 3, top) showed very good correlation with experimental measurements, with a best-fit line that was very close to $y=x$ (Exp. strain $=$ FE strain). Similar correlation coefficients were obtained for all of the sensitivity study models. Models representing standard deviations in average trabecular elastic modulus were consistently soft and had best-fit lines similar to one another (Fig 3, middle).

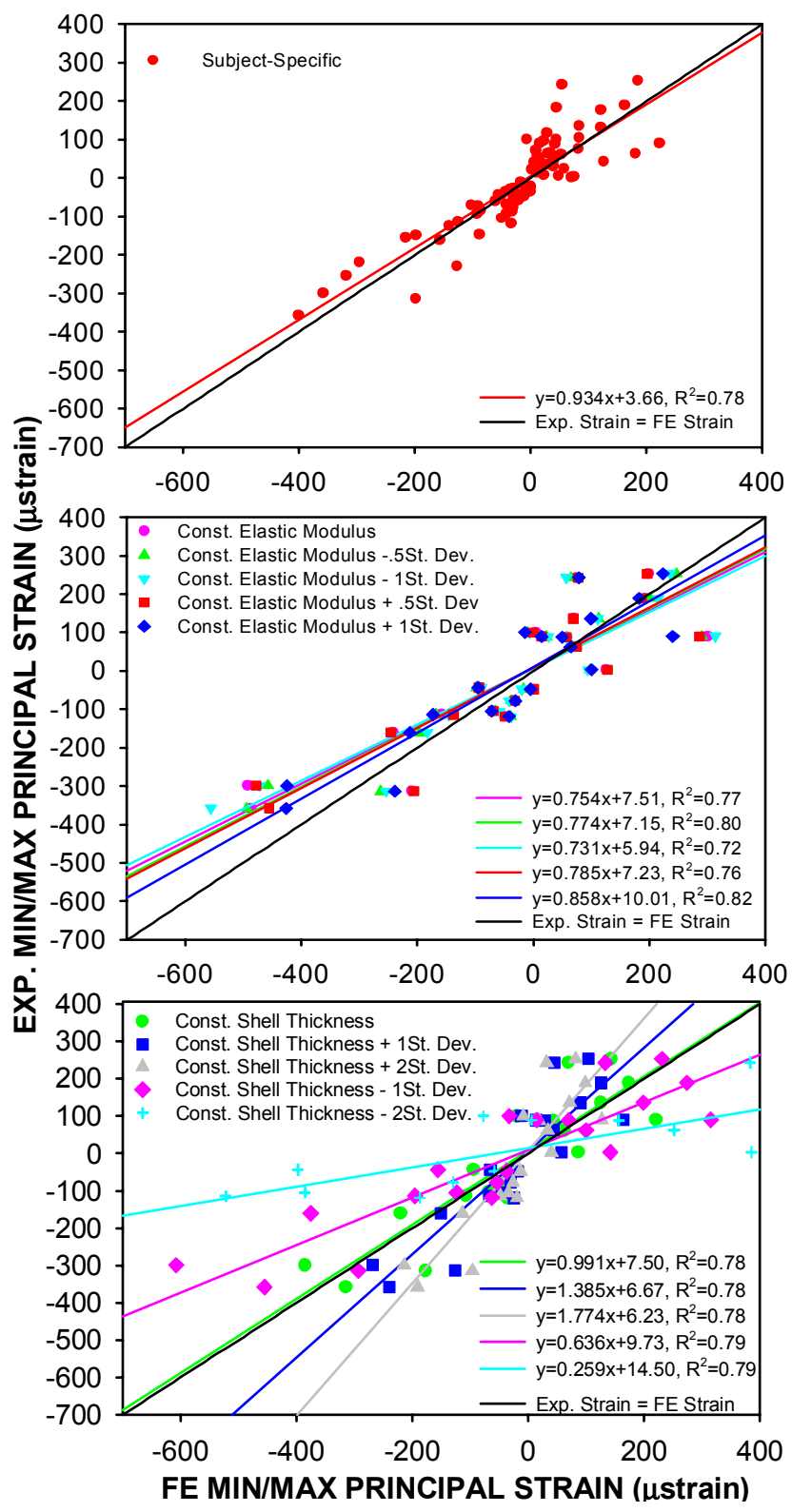

Fig 3: FE predicted vs. experimental cortical bone principal strains. Top - subject-specific, middle constant trabecular modulus, and bottom - constant cortical thickness. Data only shown for $1 \mathrm{xBW}$.
In contrast, changes in cortex thickness had a substantial effect on cortical strains. The model with average cortical thickness predicted strains that were consistent with experimental values (Fig 3, bottom). When both average thickness and trabecular elastic modulus were used, predicted strains were very close to those measured experimentally $\left(y=0.998 x+5.14, R^{2}=0.77\right.$, data not shown). The subject-specific model was too soft when the cortical Poisson's ratio was decreased to 0.2 and when the trabecular Poisson's ratio was increased to $0.29\left(y=0.730 x+20.13, R^{2}=0.81 ; y=0.788 x+7.54, R^{2}=0.81\right.$, respectively, data not shown). However, when a cortical Poisson's ratio of 0.39 was assumed, strains did not differ from the initial subject-specific model $\left(y=0.930 x+3.31, R^{2}=0.80\right.$, data not shown $)$. When the cortical elastic modulus was decreased, model predictions were too soft $\left(y=0.689 x+7.2, R^{2}=0.76\right)$, but predictions were largely unaffected when the elastic modulus was increased by one standard deviation $\left(y=0.850 x+7.10, R^{2}=0.81\right)$ (data not shown).

\section{DISCUSSION}

This research validated a subject-specific FE model of the pelvis. Accurate FE model predictions were obtained when positiondependent cortical thickness and elastic modulus were used. It was demonstrated that the FE models were most sensitive to alterations in cortical bone thickness. However, accurate predictions of cortical strains were also obtained when an average cortical thickness was used. This demonstrates that an average thickness can be used, although accurate measurement of this property should be considered.

Changes in trabecular bone elastic modulus had little effect on FE model predictions. This suggests that accurate measurement of this property is not as crucial as cortical thickness. Finally, models examining deviations in cortical elastic modulus and Poisson's ratios predicted strains that were not as accurate as the initial model, which indicates that values initially assumed were best suited for this model. The results of this study will provide the basis for future efforts to analyze patient-specific FE models of the pelvis to elucidate the biomechanics of hip dysplasia and total hip reconstruction.

\section{ACKNOWLEDGMENT}

University of Utah Funding Incentive Seed Grant.

\section{REFERENCES}

Dalstra, M., Huiskes, R., and van Erning, L., 1995, “Development and validation of a three-dimensional finite element model of the pelvic bone." J Biomech Eng, Vol 117, pp. 272-8.

Lappi, V.G., King, M.S., and Lemay, I., 1979, "Determination of elastic constants for human femurs." J Biomech Eng, Vol 101, pp. 193-197.

Little, R.B., Wevers, H. W., Siu, D., and Cooke, T. D., 1986, “A three-dimensional finite element analysis of the upper tibia." $J$ Biomech Eng, Vol 108, pp. 111-9.

Oonishi, H., Isha, T., and Hasegawa, T., 1983, "Mechanical analysis of the human pelvis and its application to the artificial hip joint-by means of the three dimensional finite element method." $J$ Biomech, Vol 16, pp. 427-44.

Ploeg, H., Taylor W., Warner M., Hertig D., and Clift S., 2001, "Finite element analysis and bone remodeling following total hip replacement." NAFEMS World Congress, Vol 2, pp. 811-822.

Snyder, S.M. and Schneider, E., 1991, "Estimation of mechanical properties of cortical bone by computed tomography." J Orthop Res, Vol 9, pp. 422-31. 